\title{
Review Article \\ Vascular Functions and Brain Integrity in Midlife: Effects of Obesity and Metabolic Syndrome
}

\author{
Andreana P. Haley ${ }^{1,2}$ \\ ${ }^{1}$ Departments of Psychology, The University of Texas at Austin, 108 E. Dean Keeton, Stop A8000, Austin, TX 78712, USA \\ ${ }^{2}$ Imaging Research Center, The University of Texas at Austin, 100 East 24th Street, Stop R9975, Austin, TX 78712, USA \\ Correspondence should be addressed to Andreana P. Haley; haley@austin.utexas.edu
}

Received 3 February 2014; Revised 25 May 2014; Accepted 4 June 2014; Published 24 June 2014

Academic Editor: David Tanne

Copyright (c) 2014 Andreana P. Haley. This is an open access article distributed under the Creative Commons Attribution License, which permits unrestricted use, distribution, and reproduction in any medium, provided the original work is properly cited.

\begin{abstract}
Intact cognitive function is the best predictor of quality of life and functional ability in older age. Thus, preventing cognitive decline is central to any effort to guarantee successful aging for our growing population of elderly. The purpose of the work discussed in this outlook paper is to bridge knowledge from basic and clinical neuroscience with the aim of improving how we understand, predict, and treat age- and disease-related cognitive impairment. Over the past six years, our research team has focused on intermediate neuroimaging phenotypes of brain vulnerability in midlife and isolating the underlying physiological mechanisms. The ultimate goal of this work was to pave the road for the development of early interventions to enhance cognitive function and preserve brain integrity throughout the lifespan.
\end{abstract}

\section{Introduction}

The most rapidly rising threat to brain health in US adults is the clustering of obesity, high blood pressure, elevated fasting glucose, and abnormal lipid metabolism in a single individual, a condition known as metabolic syndrome. A staggering $34-45 \%$ of US adults currently fulfill criteria for metabolic syndrome [1]. These numbers are alarming as metabolic syndrome is associated not only with increased risk for cardiovascular disease and diabetes [2], but also with current cognitive dysfunction and risk for future cognitive decline, over and above the detrimental effects of its components [3-10].

While we have some information about each of the disrupted peripheral physiological mechanisms in turn, very little is known about the central mechanisms that connect metabolic syndrome to brain health and cognition. The goal of our work over the past six years has been to explore the underlying neural mechanisms of midlife brain vulnerability related to peripheral vascular and metabolic disturbances, before clinically significant and permanent cognitive dysfunction has developed. Understanding the preclinical stages of disease has the enormous advantage of presenting opportunities for early intervention, a task with much higher prospect of success than attempting to restore lost function later in life. Early identification of brain vulnerability is crucial; yet it presents a significant challenge due to the low sensitivity of clinical paper-and-pencil measures of cognitive impairment and lack of norms for tests with higher ceiling performance values. Our team has endeavored to solve this problem through combining sophisticated behavioral analyses with modern neuroimaging techniques.

\section{Early Markers of Brain Vulnerability}

As noted above, one of the biggest challenges of research and clinical practice involving cognitive impairment is early detection. By the time patients experience problems with activities of daily living severe enough to prompt a visit to a health care professional and warrant a diagnosis, they are likely to have been accumulating neuropathology for decades. At this advanced stage, their brains are likely to be substantially and irreversibly damaged. Awareness of the significance of family history, early cognitive testing, genetic tests, and structural imaging have improved early detection of mild cognitive impairment, but there is still a great need 


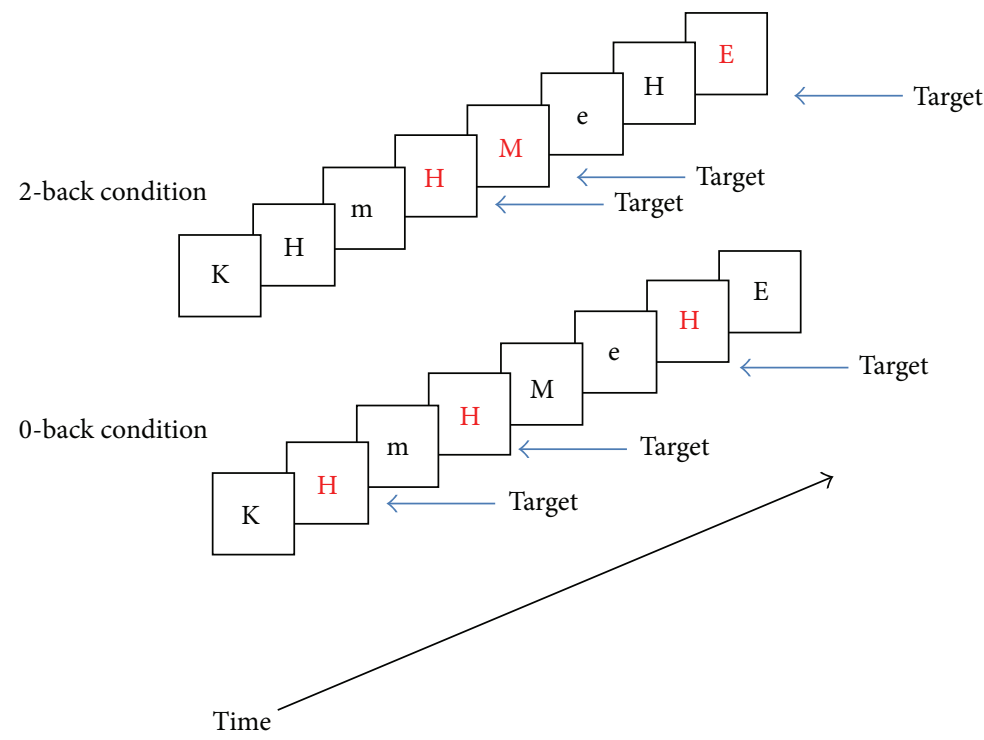

FIGURE 1: Schematic representation of the 2-back verbal working memory task. During this task, consecutive screens with letters on them appear on a computer screen for $0.5 \mathrm{~s}$. The participant is allowed for $2.5 \mathrm{~s}$ to make a response using a finger press. In the control (0-back) condition, the participant is asked to decide if the letter on the screen is the letter H. During the experimental (2-back) condition, the participant is asked to decide if the letter on the screen is the same as the letter that appeared 2 letters before, thus engaging the working memory system.

for biological markers of early brain vulnerability. Functional magnetic resonance imaging ( $\mathrm{PMRI}$ ) could be helpful in this regard. fMRI has proved to be extremely useful in identifying abnormal brain function in individuals with a variety of disorders before clinical symptoms manifest [1519]. Since this noninvasive tool can map subtle alterations in cerebral blood flow in response to changes in regional energy demands related to neuronal function [20], it has the potential to detect early signs of brain reorganization in response to developing neuropathology. Increased activation in expected task-related brain regions in at-risk groups [15, $17,19]$, bilateral recruitment of brain areas for the successful completion of normally lateralized tasks [21], and decreased potentially distracting activation (e.g., in areas devoted to self-monitoring) $[22,23]$ have all been proposed as potential signs of compensation for diminishing brain function. Coupled with well-targeted behavioral challenges that unmask early cognitive inefficiencies and compensation, fMRI can be invaluable for timely identification of obesity-related brain vulnerability as well. As evidenced by the widely used exercise stress test, dysfunction in physiological systems is more likely to manifest when the system is taxed. Thus, we have used functional brain response to a cognitive challenge as an intermediate phenotype of early brain vulnerability in many of our studies [11, 13, 24-26]. We chose a verbal working memory 2-back task (Figure 1). During this task, participants view consecutive screens with letters on them, with each letter being displayed for half a second. After the letter has disappeared from the screen, participants have two and a half seconds to make a decision whether the stimulus on the screen matches a predesignated letter. During the control condition of the task, participants are asked to decide if the letter that just flashed on the screen is the letter " $H$." During the experimental/working memory condition of the task, the participants are asked if the letter that just flashed on the screen was the same as the one that appeared two screens before. Responses are made using a two-button response box and both reaction time and response accuracy are recorded. Successful completion of this task requires intact visual function, attention, executive function, and auditory rehearsal and brain regions traditionally activated during successful completion of the task include bilateral frontal cortex, bilateral parietal lobe regions, and supplementary motor areas [27]. We chose this task as the cognitive challenge in our studies because working memory is known to activate brain areas susceptible to damage in metabolic syndrome [28] as well as cardiovascular diseases such as hypertension and coronary artery disease [29].

In search of neuroimaging markers of early brain vulnerability, we conducted several studies examining the relationships between peripheral vascular and metabolic health, working memory performance (reaction time and accuracy), and hemodynamic response and a working memory challenge as measured by fMRI. In those studies, we invited community participants between the ages of 40 and 60 to undertake neuropsychological assessment, a brain scan, and health assessment examining their body mass index (BMI), resting blood pressure, fasting lipid and glucose levels, insulin sensitivity, and measures of peripheral endothelial function such as brachial artery flow-mediated dilatation. The final study samples were diverse (less than $60 \%$ non-Hispanic Caucasian), well educated (average education level = 15 years), and cognitively intact (average Mini-Mental State Examination score, MMSE > 26 out of 30). We found that the intensity 


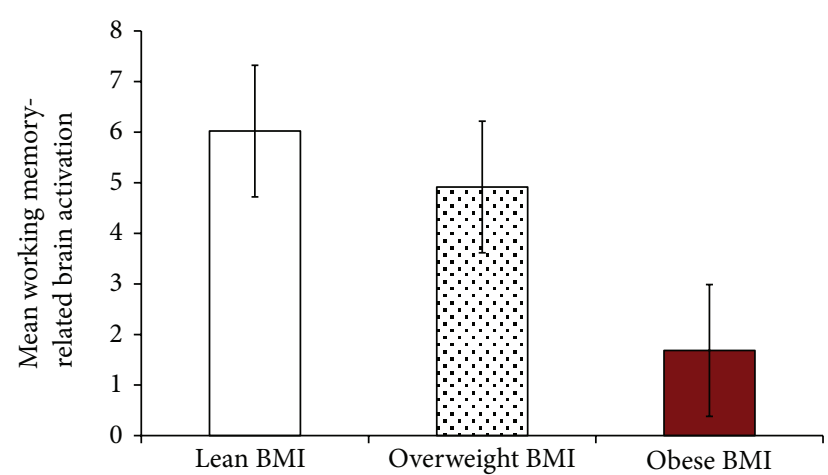

FIGURE 2: Working memory (2-back) task-related brain activation differences in right parietal cortex among otherwise healthy lean, overweight, and obese adults.

of working memory-related brain activation diminished with the development of metabolic and cardiovascular disorders as early as midlife, even when cognitive performance was still within normal limits [11, 12, 22, 25, 30]. These disorders included obesity, hypertension, metabolic syndrome, and frank cardiovascular diseases. Representative working memory task-related activation differences in middle age based on BMI are displayed in Figure 2. Despite the intact accuracy and reaction times, we interpreted the diminished task-related brain activation to indicate early brain vulnerability for two reasons: (1) in younger individuals, successful n-back task performance results in stable positive activation of the same brain regions with activation intensity increasing linearly with working memory load [31]; and (2) in older patients with cardiovascular disease, lower brain activation of these regions is related to higher level of peripheral atherosclerosis and poorer task performance [30].

In addition to fMRI, we have utilized neurospectroscopy to characterize the changes in neuronal viability and metabolism associated with metabolic and cardiovascular fitness in midlife. Proton magnetic resonance spectroscopy $\left({ }^{1} \mathrm{H}\right.$ MRS) is another safe and noninvasive magnetic resonance imaging modality with potential to enhance our ability to detect and characterize the very early stages of brain vulnerability. It utilizes the same equipment as fMRI and does not require the use of specialized coils or contrast agents. ${ }^{1} \mathrm{H}$ MRS capitalizes on the unique resonance frequencies of various hydrogen-containing compounds and prior knowledge databases to extract information about the concentration of brain metabolites of neurobiological significance from targeted brain regions. For example, one could choose a small volume of tissue (2 cc or larger) anywhere in the brain and measure the levels of $\mathrm{N}$-acetylaspartate, a marker of neuronal viability, creatine and phosphocreatine, indicators of brain energy metabolism, choline and phosphocholine, products integral to membrane turnover, myo-inositol, an organic osmolyte and precursor for the second messenger inositol triphosphate, and glutamate, the brain's major excitatory neurotransmitter [32-34]. Decreased levels of $\mathrm{N}$-acetylaspartate and increased levels of myo-inositol have been consistently reported in neurological disorders associated with cognitive dysfunction such as Down's syndrome, Alzheimer's disease, and amnestic mild cognitive impairment $[35,36]$. Alterations in glutamate levels, on the other hand, have been reported in epilepsy [37] and hypoxic-ischemic encephalopathy [38]. While neurochemical alterations in the later stages of decidedly neurodegenerative disorders may be unsurprising, work from our lab as well as others has demonstrated subtle changes in brain metabolism even in conditions not primarily known for their deleterious brain/cognitive outcomes. Our published papers have described lower brain levels of the neuronal marker $\mathrm{N}$-acetylaspartate in midlife, in relation to higher burden of peripheral atherosclerosis [39]; altered cerebral metabolism of the organic osmolyte myo-inositol in response to chronic inflammation [40]; and increased, potentially neurotoxic, cerebral levels of the excitatory neurotransmitter glutamate in middle-aged adults with metabolic syndrome [41, 42]. These findings are highly reminiscent of the prodromal stages of disorders of cognitive function such as amnestic mild cognitive impairment and Alzheimer's disease [36]. Taken together, our results indicate that even small decrements of metabolic and vascular health can have harmful brain effects and contribute to future cognitive decline. They also make a strong argument for controlling cardiovascular risk factors early in life to ensure successful cognitive as well as cardiovascular aging. Finally, they provide evidence that fMRI and neurospectroscopy can be used to develop early markers of brain vulnerability to be used in longitudinal tracking of risk as well as in the assessment of the potential efficacy of early interventions.

\section{Vascular and Nonvascular Mechanisms of Cognitive Vulnerability}

A practical challenge with the use of fMRI among asymptomatic at-risk individuals is the interpretation of the direction of blood oxygen-level-dependent (BOLD) response changes in the brain. Lower brain activity in expected taskrelated brain regions [11, 12, 24, 25], greater brain activity in expected task-related brain regions $[15,17,19]$, significant recruitment of unexpected brain regions [43, 44], and suspension of unrelated brain activity $[22,23]$ have all been reported among normally performing at-risk individuals. Ubiquitous interpretations of diminished BOLD response to a cognitive challenge as both cognitive efficiency and impairment have limited our ability to extrapolate mechanisms through interpretations of isolated fMRI findings. We have tackled this challenge by including a battery of fMRI tasks to test whether detected alterations in cerebrovascular response to a cognitive challenge are specific to brain areas related to the challenge of interest or are related to heretoforeunsuspected early global changes in cerebrovascular reactivity. Our research has shown, for example, that a breathhold calibration (i.e., consideration of the BOLD response independent of cognitive or sensory demands) increases detection of cognitive-related BOLD response differences among adults with varying levels of cardiorespiratory fitness [45]. 


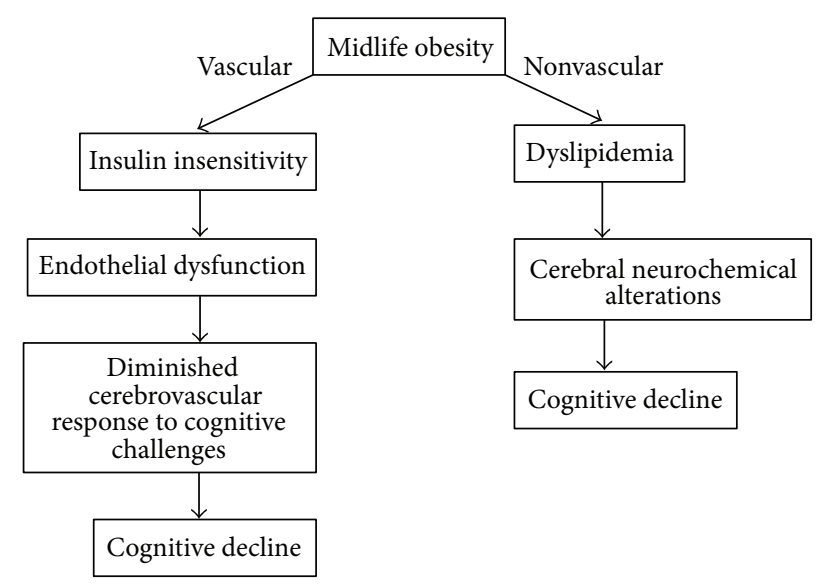

Figure 3: Proposed vascular [11, 12] and nonvascular mechanisms $[13,14]$ linking midlife obesity to poorer cognitive function in late life.

In addition, we have explored the underlying physiological mechanisms of obesity-related functional alterations by relating them to measures of cerebral metabolism as well as measures of peripheral physiological fitness. Our work demonstrates that obesity/metabolic syndrome may lead to cognitive decline through two potential pathways (Figure 3): a vascular pathway whereby obesity promotes the development of insulin resistance, which, in turn, interferes with cerebral endothelial function, leading to unfavorable alterations in the cerebrovascular response to a mental challenge and eventually to cognitive decline [11, 12]. To test this model, we conducted a study exploring the relationship between BMI, insulin sensitivity, and cerebrovascular response and a cognitive challenge using fMRI. We used the working memory 2-back task described earlier. As in the previous studies, middle-aged, otherwise healthy, ethnically diverse, and cognitively intact community volunteers participated in this study. The results showed a dose-dependent relationship between BMI and task-related functional brain activation in the right parietal cortex with lean adults (BMI $<25$ ) exhibiting higher task-related brain activation than overweight $(25<\mathrm{BMI}<30)$ and obese $(\mathrm{BMI} \geq 30)$ adults (Figure 2) [12]. More importantly, this relationship appeared to be fully accounted for by insulin sensitivity, providing supporting evidence for our vascular model of obesityrelated brain vulnerability. Yet, the model hypothesized that insulin sensitivity impacts the hemodynamic response to a cognitive challenge by contributing to endothelial dysfunction, assuming a relationship between endothelial function and task-related functional brain activation. To test this assumption, we conducted another study on communitydwelling middle-aged adults during which we measured functional brain response to a working memory challenge using fMRI and brachial artery endothelial-dependent flowmediated dilation (FMD) using B-mode ultrasound [12]. We established that there was a significant positive correlation between FMD and task-related brain activation in the right superior parietal cortex where greater FMD was related to a more robust cerebrovascular response to the cognitive challenge. Thus, we found supporting evidence for all of the critical steps of the proposed vascular model of obesityrelated brain vulnerability where obesity, through the development of insulin insensitivity, was hypothesized to lead to endothelial dysfunction and eventually to lower task-related functional brain activation and cognitive decline.

However, unsurprising for such a complex metabolic condition, we also found evidence for a nonvascular pathway via which obesity could also lead to disturbances in cognitive function. Again, our studies included middle-aged community volunteers (aged from 40 to 60), free of major neurological and psychiatric disorders, ethnically diverse $(<45 \%$ non-Hispanic Caucasian), well educated (average education level =15), and cognitively intact (MMSE > 27 out of 30). They underwent a neuropsychological evaluation, a health screen, and structural brain imaging including neurospectroscopy. We found that BMI was negatively correlated with memory performance with participants with higher BMI performing worse on a word-learning task. While this relationship was only a trend in midlife, a significant indirect effect of cerebral myo-inositol was detectable. Higher BMI was related to poorer memory performance in midlife through elevated cerebral myo-inositol [13]. In search for a modifiable risk factor for cognitive impairment, we continued to probe the BMI-myo-inositol relationship further, discovering that it was fully accounted for by elevated triglycerides and reduced levels of high-density lipoprotein (HDL) cholesterol [14]. Together, these studies provide evidence that obesity-related disturbance of lipid metabolism may directly affect cerebral neurochemistry by altering fluid homeostasis and promoting neuroinflammation, again, resulting in deleterious cognitive effects $[13,14]$. Since the ultimate goal of all of this work is to identify viable targets for early interventions to promote successful cognitive aging, these latest findings are of particular significance as both insulin resistance and disorders of lipid metabolism are highly treatable.

\section{Future Direction 1: Interventions to Preserve Brain Function}

Exercise Interventions. Following the vascular model of obesity-related brain vulnerability, one highly promising intervention to preserve brain function is aerobic exercise. The cardiovascular benefits of aerobic exercise are well known [46] and fitness training has been associated with cognitive enhancement in nondemented older adults [47]. Our own research has demonstrated that fitness-related benefits on neuronal viability [48], cerebral perfusion [49], and cognitive function [50] are evident as early as midlife. However, it is still unclear whether this benefit is simply due to prevention of other risk factors (e.g., reductions in blood pressure), or whether exercise can reverse the negative effects of aging on the brain. Future work should employ sensitive neuroimaging measures of neuronal integrity, cerebral perfusion, and cognition to test the effects of exercise training in previously sedentary middle-aged adults. 
Nonexercise Interventions. An ideal intervention for patients with any kind of cardiovascular disease would involve restoring the original cardiovascular status, through aerobic exercise, for example. As this is often not feasible (only 5\% of US adults report the recommended level of daily physical activity [51]), an alternative intervention would be to support brain energy production by upregulating mitochondrial respiration [52]. One highly promising novel intervention to stimulate brain energy production is transcranial low-level light/laser therapy (LLLT), the use of directional low-power and highfluence monochromatic or quasimonochromatic light from lasers or light-emitting diodes in the red to near-infrared wavelengths [53]. This intervention capitalizes on the fact that the brain is capable of converting light to energy stored as adenosine triphosphate (ATP), the energy used by all metabolic processes. The conversion is facilitated by the mitochondrial enzyme cytochrome oxidase. Transcranial LLLT utilizes the ability of cytochrome oxidase to accept photons from near-infrared light in the brain. Thus, transcranial LLLT is intended to modify mitochondrial respiration in the brain, rather than alter vascular function [54]. LLLT stimulates ATP production by upregulating cytochrome oxidase activity [54]. It is safe, noninvasive, and therapeutically beneficial, promoting enhancement of energy production, gene expression, and prevention of cell death. It has been FDA-approved for pain relief in head, neck, and hand [55]. Very recently, it was demonstrated that even a single LLLT treatment with a laser diode (CG-5000) to the forehead improves both mood and cognitive function in healthy young adults [56]. Forty volunteers, aged from 18 to 35 , were randomized into one of two treatment conditions: LLLT or control. In the active condition, participants received four 1-minute LLLT treatments to each of two sites on the right forehead during a single treatment session. In the control condition, participants experienced a similar protocol with the only difference being that, within each 1-minute active treatment period, they received 5 seconds of LLLT and 55 seconds of no treatment. Sustained attention, memory, and mood were measured before and after treatment using psychomotor vigilance and delayed match-to-sample tasks as well as selfreport measures of positive and negative affect (Positive and Negative Affect Schedule (PANAS)). While no differences were noted between the groups on any of the measures before treatment, reaction time on the psychomotor vigilance task, memory retrieval latency, and correct responses after treatment were significantly better in the actively treated participants as compared to the controls. Overall affect, calculated as positive affect score minus negative affect score, was also significantly higher after treatment in the actively treated participants. The authors concluded that these results provide supporting evidence for the efficacy of LLLT in improving cognitive performance as well as mood. Since the frontoparietal networks responsible for sustained attention appear to be particularly vulnerable to damage related to cardiovascular and metabolic disorders [29], LLLT may be a viable treatment alternative in those patient populations as well, especially in cases where restoring the original cardiovascular status is not feasible. Future studies should extend knowledge in this area by examining the effects of LLLT in middle-aged and older participants at varying degrees of risk for developing cardiovascular and cerebrovascular disorders including vascular cognitive impairment.

\section{Future Direction 2: Moderators of Cognitive Vulnerability}

While a great wealth of knowledge about early brain vulnerability has resulted from studies of group differences between people with cognitive risk factors and their healthy counterparts, it has also become increasingly clear that the cognitive trajectories of individuals with the same set of risk factors may vary. This latter fact brings to light the complex interactions between genetic susceptibilities and acquired environmental risk factors in conferring both risk and resilience. Future studies should examine the role of genetic (e.g., ApoE4) and environmental moderators of cognitive vulnerability (e.g., psychosocial stress) and resilience (e.g., education). Special attention should be devoted to health promotion among underserved populations such as individuals with lower socioeconomic status and migrant workers.

\section{Summary and Conclusions}

In summary, the goal of our work has been to explore the underlying neural mechanisms of brain vulnerability related to metabolic syndrome and subclinical cardiovascular disease, before brain function is significantly compromised. To accomplish this, we have relied on a combination of behavioral challenges and cutting-edge technologies including functional magnetic resonance imaging, neurospectroscopy, and ultrasonography. The intermediate neuroimaging phenotypes of early brain vulnerability developed in our lab, in addition to information about the neurochemical changes underlying any functional alterations, offer opportunities to pilot the efficacy of early exercise and nonexercise interventions to promote successful cognitive aging. Future studies should also include the systematic assessment of genetic (e.g., ApoE4) and environmental moderators of cognitive vulnerability (e.g., psychosocial stress) and resilience (e.g., education). Special attention should be paid to health promotion among underserved populations such as individuals with lower socioeconomic status and migrant workers.

\section{Conflict of Interests}

The author declares that there is no conflict of interests regarding the publication of this paper.

\section{Acknowledgments}

This work was made possible in part by funding provided by the American Heart Association (09BGIA2060722), the American Federation for Aging Research (8A0024), the National Institute of Neurological Disorders and Stroke (R01 NS075565), and the University of Texas at Austin. 


\section{References}

[1] R. B. Ervin, "Prevalence of metabolic syndrome among adults 20 years of age and over, by sex, age, race and ethnicity, and body mass index: United States, 2003-2006," National Health Statistics Reports, no. 13, pp. 1-7, 2009.

[2] K. G. M. M. Alberti, R. H. Eckel, S. M. Grundy et al., "Harmonizing the metabolic syndrome: A joint interim statement of the international diabetes federation task force on epidemiology and prevention; National heart, lung, and blood institute; American heart association; World heart federation; International atherosclerosis society; And international association for the study of obesity," Circulation, vol. 120, no. 16, pp. 1640-1645, 2009.

[3] T. N. Akbaraly, M. Kivimaki, M. J. Shipley et al., "Metabolic syndrome over 10 years and cognitive functioning in late midlife: the Whitehall II study," Diabetes Care, vol. 33, no. 1, pp. 84-89, 2010.

[4] S. Kalmijn, D. Foley, L. White et al., "Metabolic cardiovascular syndrome and risk of dementia in Japanese-American elderly men: the Honolulu-Asia aging study," Arteriosclerosis, Thrombosis, and Vascular Biology, vol. 20, no. 10, pp. 2255-2260, 2000.

[5] K. Yaffe, M. Haan, T. Blackwell, E. Cherkasova, R. A. Whitmer, and N. West, "Metabolic syndrome and cognitive decline in elderly latinos: findings from the Sacramento Area Latino Study of Aging Study," Journal of the American Geriatrics Society, vol. 55, no. 5, pp. 758-762, 2007.

[6] K. Yaffe, A. L. Weston, T. Blackwell, and K. A. Krueger, "The metabolic syndrome and development of cognitive impairment among older women," Archives of Neurology, vol. 66, no. 3, pp. 324-328, 2009.

[7] N. M. Gatto, V. W. Henderson, J. A. St. John, C. McCleary, H. N. Hodis, and W. J. Mack, "Metabolic syndrome and cognitive function in healthy middle-aged and older adults without diabetes," Aging, Neuropsychology, and Cognition, vol. 15, no. 5, pp. 627-641, 2008.

[8] M. G. Dik, C. Jonker, H. C. Comijs et al., "Contribution of metabolic syndrome components to cognition in older individuals," Diabetes Care, vol. 30, no. 10, pp. 2655-2660, 2007.

[9] M. Vanhanen, K. Koivisto, L. Moilanen et al., "Association of metabolic syndrome with Alzheimer disease: a populationbased study," Neurology, vol. 67, no. 5, pp. 843-847, 2006.

[10] C. Raffaitin, H. Gin, J.-P. Empana et al., "Metabolic syndrome and risk for incident alzheimer's disease or vascular dementia," Diabetes Care, vol. 32, no. 1, pp. 169-174, 2009.

[11] M. M. Gonzales, T. Tarumi, H. Tanaka et al., "Functional imaging of working memory and peripheral endothelial function in middle-aged adults," Brain and Cognition, vol. 73, no. 2, pp. 146$151,2010$.

[12] M. M. Gonzales, T. Tarumi, S. C. Miles, H. Tanaka, F. Shah, and A. P. Haley, "Insulin sensitivity as a mediator of the relationship between BMI and working memory-related brain activation," Obesity, vol. 18, no. 11, pp. 2131-2137, 2010.

[13] M. M. Gonzales, T. Tarumi, D. E. Eagan, H. Tanaka, M. Vaghasia, and A. P. Haley, "Indirect effects of elevated body mass index on memory performance through altered cerebral metabolite concentrations," Psychosomatic Medicine, vol. 74, no. 7, pp. 691-698, 2012.

[14] A. P. Haley, M. M. Gonzales, T. Tarumi, and H. Tanaka, "Dyslipidemia links obesity to early cerebral neurochemical alterations," Obesity, vol. 21, no. 10, pp. 2007-2013, 2013.
[15] S. Y. Bookheimer, M. H. Strojwas, M. S. Cohen et al., "Patterns of brain activation in people at risk for Alzheimer's disease," New England Journal of Medicine, vol. 343, no. 7, pp. 450-456, 2000.

[16] L. Chang, O. Speck, E. N. Miller et al., "Neural correlates of attention and working memory deficits in HIV patients," Neurology, vol. 57, no. 6, pp. 1001-1007, 2001.

[17] A. J. Saykin, L. A. Flashman, S. A. Frutiger et al., "Neuroanatomic substrates of semantic memory impairment in Alzheimer's disease: patterns of functional MRI activation," Journal of the International Neuropsychological Society, vol. 5, no. 5, pp. 377-392, 1999.

[18] L. H. Sweet, S. M. Rao, M. Primeau, S. Durgerian, and R. A. Cohen, "Functional magnetic resonance imaging response to increased verbal working memory demands among patients with multiple sclerosis," Human Brain Mapping, vol. 27, no. 1, pp. 28-36, 2006.

[19] L. H. Sweet, S. M. Rao, M. Primeau, A. R. Mayer, and R. A. Cohen, "Functional magnetic resonance imaging of working memory among multiple sclerosis patients," Journal of Neuroimaging, vol. 14, no. 2, pp. 150-157, 2004.

[20] S. Ogawa, T. M. Lee, A. R. Kay, and D. W. Tank, "Brain magnetic resonance imaging with contrast dependent on blood oxygenation," Proceedings of the National Academy of Sciences of the United States of America, vol. 87, no. 24, pp. 9868-9872, 1990.

[21] R. Cabeza, N. D. Anderson, J. K. Locantore, and A. R. McIntosh, "Aging gracefully: compensatory brain activity in highperforming older adults," NeuroImage, vol. 17, no. 3, pp. 1394$1402,2002$.

[22] A. P. Haley, J. Gunstad, R. A. Cohen, B. A. Jerskey, R. C. Mulligan, and L. H. Sweet, "Neural correlates of visuospatial working memory in healthy young adults at risk for hypertension," Brain Imaging and Behavior, vol. 2, no. 3, pp. 192-199, 2008.

[23] L. H. Sweet, J. F. Paskavitz, A. P. Haley et al., "Imaging phonological similarity effects on verbal working memory," Neuropsychologia, vol. 46, no. 4, pp. 1114-1123, 2008.

[24] M. M. Gonzales, T. Tarumi, D. E. Eagan, H. Tanaka, F. O. Biney, and A. P. Haley, "Current serum lipoprotein levels and fMRI response to working memory in midlife," Dementia and Geriatric Cognitive Disorders, vol. 31, no. 4, pp. 259-267, 2011.

[25] K. F. Hoth, M. M. Gonzales, T. Tarumi, S. C. Miles, H. Tanaka, and A. P. Haley, "Functional MR imaging evidence of altered functional activation in metabolic syndrome," The American Journal of Neuroradiology, vol. 32, no. 3, pp. 541-547, 2011.

[26] A. P. Haley, D. E. Eagan, M. M. Gonzales, F. O. Biney, and R. A. Cooper, "Functional magnetic resonance imaging of working memory reveals frontal hypoactivation in middle-aged adults with cognitive complaints," Journal of the International Neuropsychological Society, vol. 17, no. 5, pp. 915-924, 2011.

[27] T. D. Wager and E. E. Smith, "Neuroimaging studies of working memory: a meta-analysis," Cognitive, Affective and Behavioral Neuroscience, vol. 3, no. 4, pp. 255-274, 2003.

[28] B. Segura, M. Á. Jurado, N. Freixenet, C. Albuin, J. Muniesa, and C. Junqué, "Mental slowness and executive dysfunctions in patients with metabolic syndrome," Neuroscience Letters, vol. 462, no. 1, pp. 49-53, 2009.

[29] R. A. Cohen, A. Poppas, D. E. Forman et al., "Vascular and cognitive functions associated with cardiovascular disease in the elderly," Journal of Clinical and Experimental Neuropsychology, vol. 31, no. 1, pp. 96-110, 2009.

[30] A. P. Haley, L. H. Sweet, J. Gunstad et al., "Verbal working memory and atherosclerosis in patients with cardiovascular 
disease: an fMRI study," Journal of Neuroimaging, vol. 17, no. 3, pp. 227-233, 2007.

[31] J. D. Ragland, B. I. Turetsky, R. C. Gur et al., "Working memory for complex figures: an fMRI comparison of letter and fractal nback tasks," Neuropsychology, vol. 16, no. 3, pp. 370-379, 2002.

[32] E. R. Danielsen and B. Ross, Magnetic Resonance Spectroscopy Diagnosis of Neurological Diseases, Marcel Dekker, New York, NY, USA, 1999.

[33] B. D. Ross, "Biochemical considerations in $1 \mathrm{H}$ spectroscopy. Glutamate and glutamine; myo-inositol and related metabolites," NMR in Biomedicine, vol. 4, no. 2, pp. 59-63, 1991.

[34] M. Erecińska and I. A. Silver, "Metabolism and role of glutamate in mammalian brain," Progress in Neurobiology, vol. 35, no. 4, pp. 245-296, 1990.

[35] G. T. Berry, Z. J. Wang, S. F. Dreha, B. M. Finucane, and R. A. Zimmerman, "In vivo brain myo-inositol levels in children with Down syndrome," Journal of Pediatrics, vol. 135, no. 1, pp. 94-97, 1999.

[36] K. Kantarci, C. R. Jack Jr., Y. C. Xu et al., "Regional metabolic patterns in mild cognitive impairment and Alzheimer's disease: a 1H MRS study," Neurology, vol. 55, no. 2, pp. 210-217, 2000.

[37] G. Helms, C. Ciumas, S. Kyaga, and I. Savic, "Increased thalamus levels of glutamate and glutamine (Glx) in patients with idiopathic generalised epilepsy," Journal of Neurology, Neurosurgery and Psychiatry, vol. 77, no. 4, pp. 489-494, 2006.

[38] Y. Pu, Q.-F. Li, C.-M. Zeng et al., "Increased detectability of alpha brain glutamate/glutamine in neonatal hypoxic-ischemic encephalopathy," The American Journal of Neuroradiology, vol. 21, no. 1, pp. 203-212, 2000.

[39] A. P. Haley, T. Tarumi, M. M. Gonzales, J. Sugawara, and H. Tanaka, "Subclinical atherosclerosis is related to lower neuronal viability in middle-aged adults: a 1 H MRS study," Brain Research, vol. 1344, pp. 54-61, 2010.

[40] D. E. Eagan, M. M. Gonzales, T. Tarumi, H. Tanaka, S. Stautberg, and A. P. Haley, "Elevated serum C-reactive protein relates to increased cerebral myoinositol levels in middle-aged adults," Cardiovascular Psychiatry and Neurology, vol. 2012, Article ID 120540, 9 pages, 2012.

[41] A. P. Haley, M. M. Gonzales, T. Tarumi, and H. Tanaka, "Subclinical vascular disease and cerebral glutamate elevation in metabolic syndrome," Metabolic Brain Disease, vol. 27, no. 4, pp. 513-520, 2012.

[42] A. P. Haley, M. M. Gonzales, T. Tarumi, S. C. Miles, K. Goudarzi, and H. Tanaka, "Elevated cerebral glutamate and myo-inositol levels in cognitively normal middle-aged adults with metabolic syndrome," Metabolic Brain Disease, vol. 25, no. 4, pp. 397-405, 2010.

[43] I.-K. Penner, M. Rausch, L. Kappos, K. Opwis, and E. W. Radü, "Analysis of impairment related functional architecture in MS patients during performance of different attention tasks," Journal of Neurology, vol. 250, no. 4, pp. 461-472, 2003.

[44] W. Staffen, A. Mair, H. Zauner et al., "Cognitive function and fMRI in patients with multiple sclerosis: evidence for compensatory cortical activation during an attention task," Brain, vol. 125, no. 6, pp. 1275-1282, 2002.

[45] M. M. Gonzales, T. Tarumi, J. A. Mumford et al., "Greater BOLD response to working memory in endurance-trained adults revealed by breath-hold calibration," Human Brain Mapping, vol. 35, no. 7, pp. 2898-2910, 2013.

[46] G. F. Fletcher, G. Balady, S. N. Blair et al., "Statement on exercise: benefits and recommendations for physical activity programs for all Americans: a statement for health professionals by the committee on exercise and cardiac rehabilitation of the Council on Clinical Cardiology, American Heart Association," Circulation, vol. 94, no. 4, pp. 857-862, 1996.

[47] J. L. Etnier, W. Salazar, D. M. Landers, S. J. Petruzzello, M. Han, and P. Nowell, "The influence of physical fitness and exercise upon cognitive functioning: a meta-analysis," Journal of Sport and Exercise Psychology, vol. 19, no. 3, pp. 249-277, 1997.

[48] M. M. Gonzales, T. Tarumi, S. Kaur et al., "Aerobic fitness and the brain: increased $\mathrm{N}$-acetyl-aspartate and choline concentrations in endurance-trained middle-aged adults," Brain Topography, vol. 26, no. 1, pp. 126-134, 2013.

[49] T. Tarumi, M. M. Gonzales, B. Fallow et al., "Central artery stiffness, neuropsychological function, and cerebral perfusion in sedentary and endurance-trained middle-aged adults," Journal of Hypertension, vol. 31, no. 12, pp. 2400-2409, 2013.

[50] T. Tarumi, M. M. Gonzales, B. Fallow et al., "Aerobic fitness and cognitive function in midlife: an association mediated by plasma insulin," Metabolic Brain Disease, vol. 28, no. 4, pp. 727730, 2013.

[51] T. W. Buford, M. D. Roberts, and T. S. Church, "Toward exercise as personalized medicine," Sports Medicine, vol. 43, no. 3, pp. 157-165, 2013.

[52] F. Gonzalez-Lima, B. R. Barksdale, and J. C. Rojas, "Mitochondrial respiration as a target for neuroprotection and cognitive enhancement," Biochemical Pharmacology, vol. 88, no. 4, pp. 584-593, 2014.

[53] J. C. Rojas and F. Gonzalez-Lima, "Low-level light therapy of the eye and brain," Eye and Brain, vol. 3, pp. 49-67, 2011.

[54] J. C. Rojas and F. Gonzalez-Lima, "Neurological and psychological applications of transcranial lasers and LEDs," Biochemical Pharmacology, vol. 86, no. 4, pp. 447-457, 2013.

[55] A. M. Fulop, S. Dhimmer, J. R. Deluca et al., "A meta-analysis of the efficacy of laser phototherapy on pain relief," Clinical Journal of Pain, vol. 26, no. 8, pp. 729-736, 2010.

[56] D. W. Barrett and F. Gonzalez-Lima, "Transcranial infrared laser stimulation produces beneficial cognitive and emotional effects in humans," Neuroscience, vol. 230, pp. 13-23, 2013. 


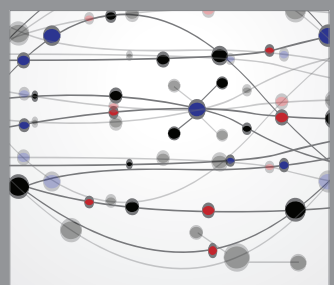

The Scientific World Journal
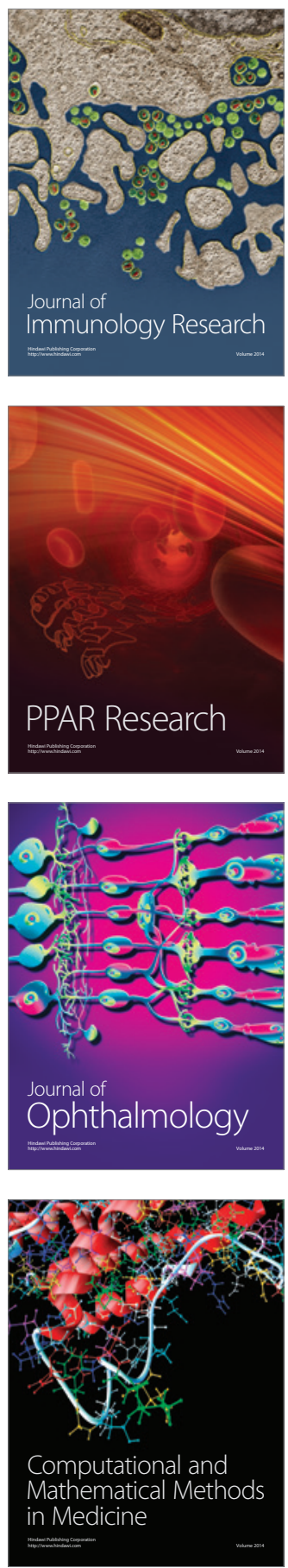

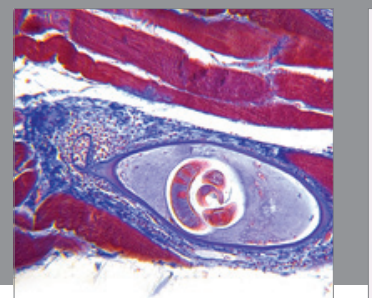

Gastroenterology

Research and Practice
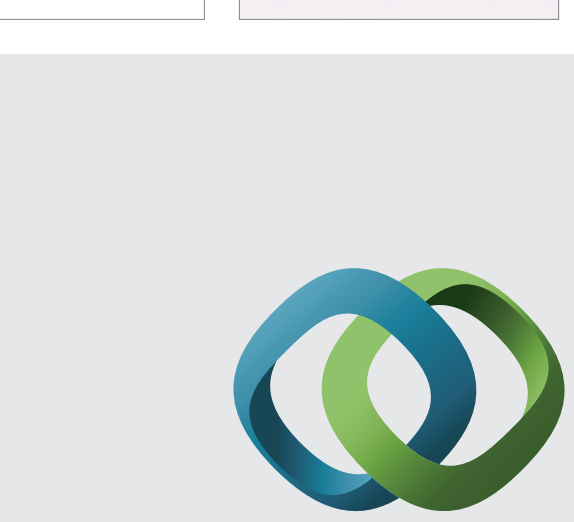

\section{Hindawi}

Submit your manuscripts at

http://www.hindawi.com
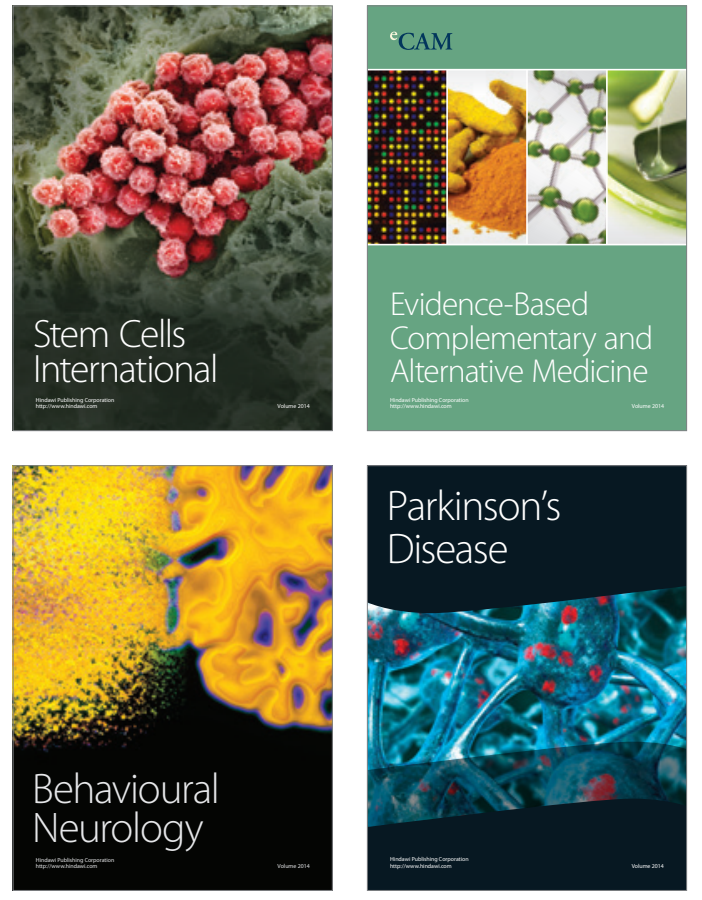
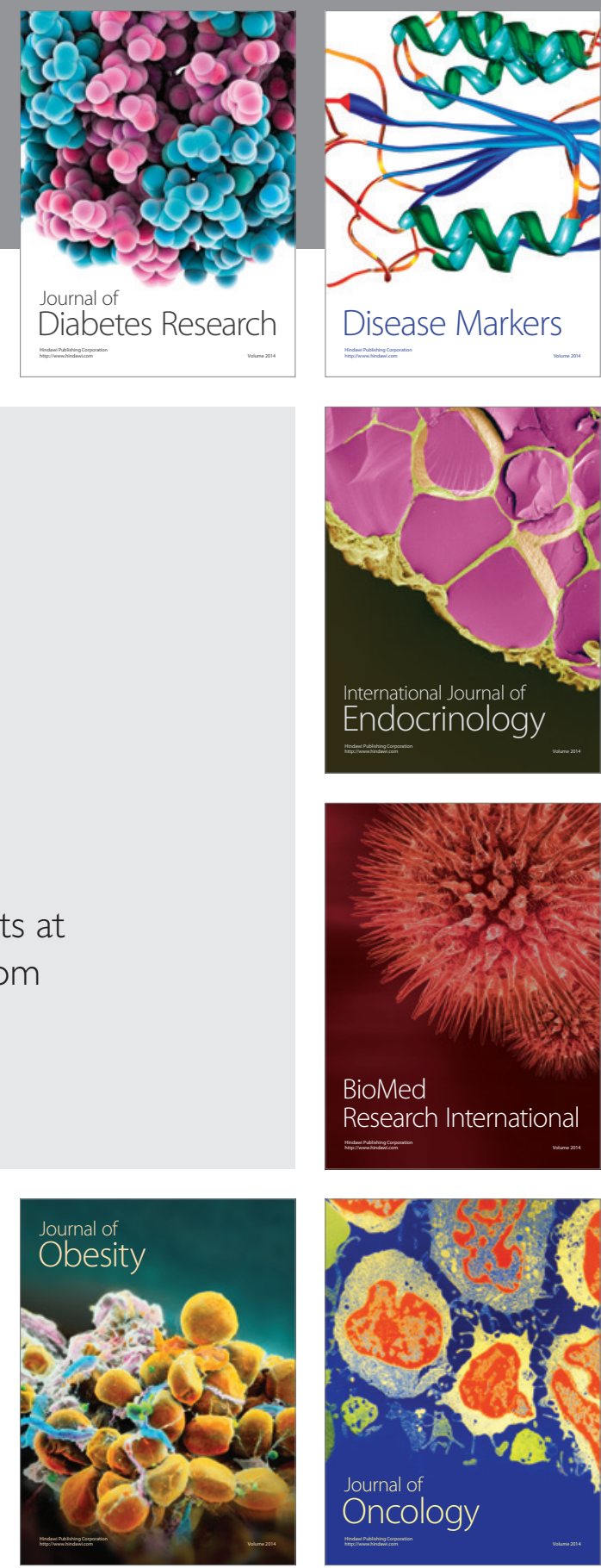

Disease Markers
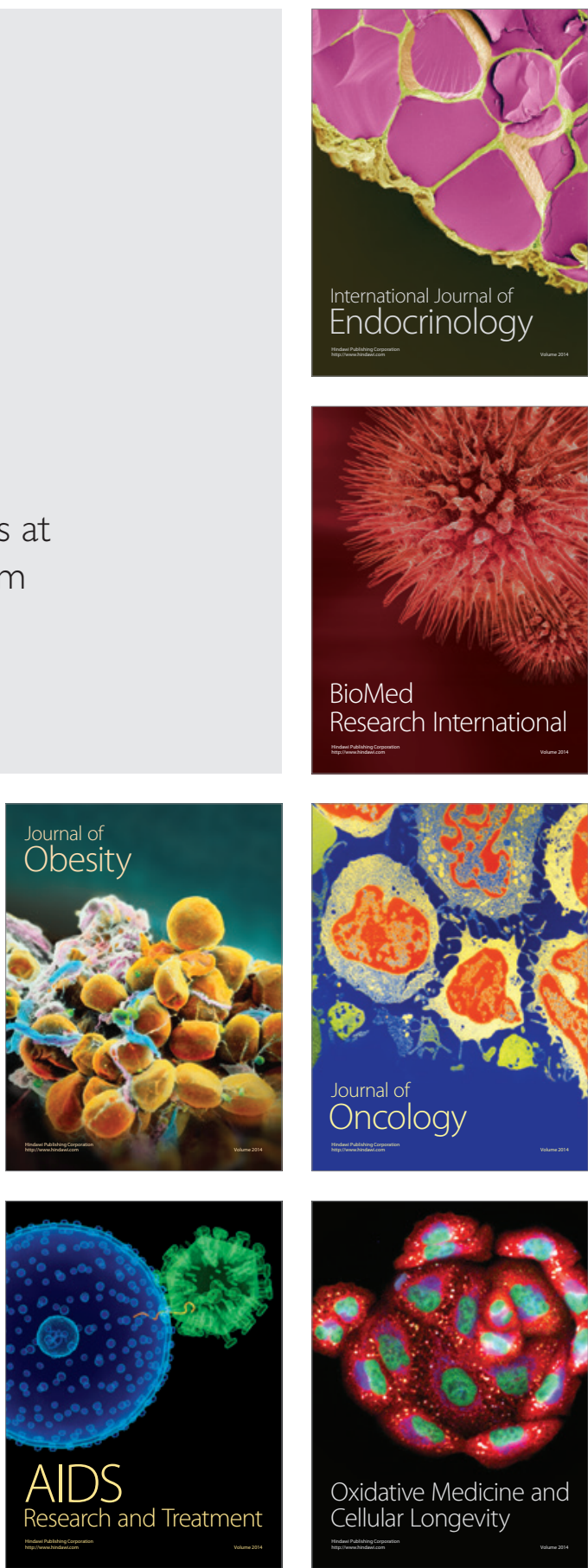